\title{
The Design of PROFIBUS-DP AI Based on Butterworth
}

\author{
Jiao Ping \\ Lab. of Networked Control Systems \\ Shenyang Institute of Automation (SIA), Chinese Academy \\ of Sciences China, Shenyang \\ jiaoping@sia.cn
}

\author{
Zhou Tong \\ Lab. of Networked Control Systems \\ Shenyang Institute of Automation (SIA), Chinese \\ Academy of Sciences China, Shenyang \\ tzhou@sia.cn
}

\begin{abstract}
For the high frequency characteristics of the signal acquisition system and the noise of industrial field, this paper proposed a design of PROFIBUS-DP AI based on multi-order Butterworth low-pass filter, and gave the derivation process of filter, calculation process of $\mathrm{RC}$ value. It has been found that this scheme can effectively improve the signal-to-noise ratio of the system in industrial field, has strong control ability to suppress interference signal, and has the advantage of smaller distortion and so on to the input signal which should be measured.
\end{abstract}

Keywords-PROFIBUS-DP; AI; Butterworth;

\section{INTRODUCTION}

PROFIBUS (Process Field Bus) is a standard for field bus communication in automation technology. The success of PROFIBUS is equally due to its advanced technology and the successful activities of the organization, which was founded to represent the interests of manufacturers and users. PROFIBUS AI is a signal acquisition system based on PROFIBUS, which converts analog signals of industrial field (Temperature, Pressure, speed, current) to digital signals, and sent to MCU[1].

The environment of industrial field is very complex, and there are plenty of noise signals which mainly high frequency signal noise. The serious high frequency signal noise would be interference the signal acquisition system which in industrial process control. In order to reduce the high frequency noise signal, the system design often adopt one order, two order of resistive capacitive filter or damper filter, but this kind filter always with low SNR, and the measured signals are attenuated to a certain extent, which is not very ideal [2-3]. The Butterworth filters have the well response performance of low pass filter characteristics and the process of control system is required, it began to be used in the design of industrial filter.

\section{BUTTERWORTH FILTER}

The Characteristics of the Butterworth filter is that the pass band frequency response curves is the maximally flat, no ups and downs, and in the stop band is gradually decreased to zero. The frequency and amplitude of some boundary, from the beginning of the angular frequency, amplitude and gradually decreased with the increase of frequency, tends to negative infinity which in figure of Potter log diagonal.
The design of analog filter is that a set of rules into the corresponding function $H_{a}(s)$, than the approximation properties of an ideal filter. Amplitude square function definitions of Butterworth low pass filter for:

$$
|H(j \lambda)|^{2}=\frac{1}{1+C^{2} \lambda^{2 N}}
$$

The $\mathrm{C}$ is a constant parameter, $\mathrm{N}$ is the filter order, as the normalized low-pass cutoff frequency, where $\mathrm{N}$ is an integer, is of order filter[4].

Butterworth low-pass filter with a maximum flat amplitude characteristics in the passband, that is to say, $\mathrm{N}$-order low-pass filter in front of ' $2 \mathrm{~N}-1$ ' -order derivative of the magnitude squared function equal to zero, approaching the stopband is monotonic change. The Figure 1 shown is Amplitude characteristics of Butterworth low-pass filter.

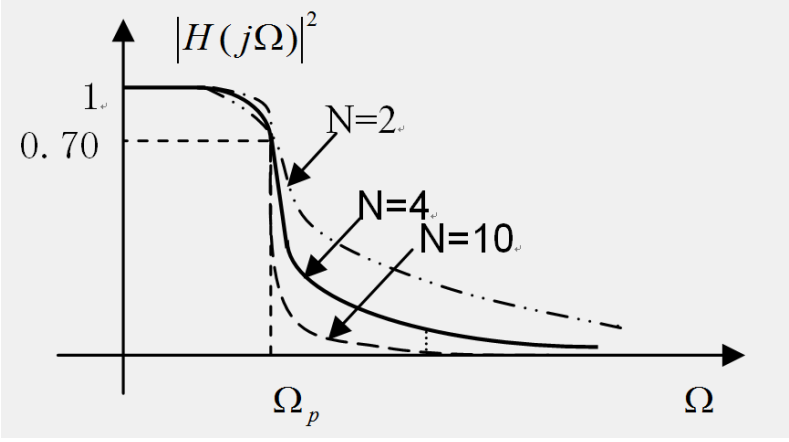

Figure 1. Amplitude characteristics of Butterworth low-pass filter

The characteristic of the filter is completely determined by the order $\mathrm{N}$. When $\mathrm{N}$ increases, the filter characteristic curve becomes steeper, though this time by the formula (1) determine the magnitude of the attenuation function in $\Omega=\Omega_{p}$ is always at $3 \mathrm{~dB}$, but they will be close in the larger context of a passband is close to 1, more rapidly in the stop band close to zero, and thus closer to the ideal characteristics of the amplitude frequency characteristic of a rectangle. Amplitude characteristics of the filter parameters of $\mathrm{N}$ dependence as shown in figure 1.

Common design Butterworth lowpass filter indicators: $\lambda_{p}$ : Cutoff frequency of Pass band; 
$\alpha_{p}$ : Attenuation of Pass band, unit: $\mathrm{dB}$.

$\lambda_{s}$ : Starting frequency of Stopband;

$\alpha_{s}$ : Attenuation of Stopband, unit: $\mathrm{dB}$.

Notes:

1) The attenuation in decibels $(d B)$ as a unit; i.e.

$$
\alpha=10 \lg \frac{1}{|H(j \lambda)|^{2}}=10 \lg \left[1+C^{2} \lambda^{2 N}\right]
$$

2) As $\quad \alpha=3 d B$, the $\Omega_{p}=\Omega_{C}$ is the cutoff frequency.

3) In the filter design often chosen normalized frequency $\lambda=\Omega / \Omega_{C}$ :

$$
\lambda_{p}=\frac{\Omega_{p}}{\Omega_{p}}=1, \quad \lambda_{s}=\frac{\Omega_{s}}{\Omega_{p}}
$$

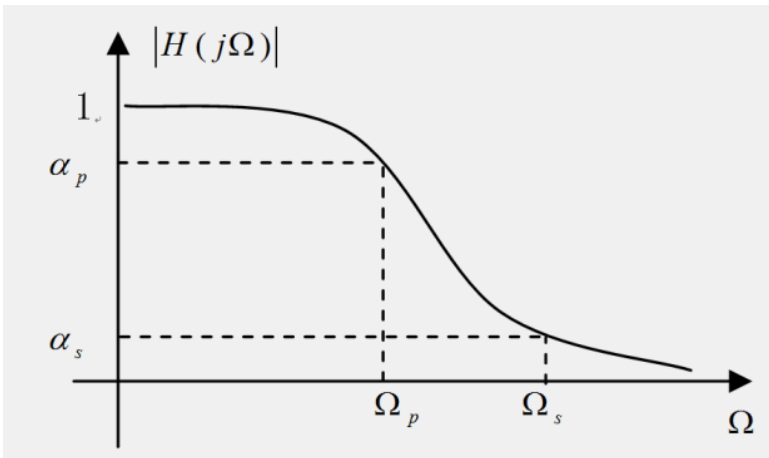

Figure 2. The parameters of Butterworth low pass filter

According to the design requirements of $\lambda_{p}, \alpha_{p}, \lambda_{s}$, $\alpha_{s}$, determine undetermined coefficients $\mathrm{C}$ and filter order $\mathrm{N}$ of the normalized Butterworth low-pass filter magnitude squared function, then according to the amplitude square function to determine the transfer function $\mathrm{H}(\mathrm{s})$ Butterworth low pass filter[5-6].

The actual frequency $\Omega$ after normalized the $\lambda_{p}=\frac{\Omega_{p}}{\Omega_{p}}=1$ and $\lambda_{s}=\frac{\Omega_{s}}{\Omega_{p}}$. Then according to the known ' $\alpha_{p}$ ' ' $\alpha_{s}$ ', and amplitude square function $|H(j \lambda)|^{2}=\frac{1}{1+C^{2} \lambda^{2 N}} \quad$, calculated the parameter $\mathrm{C}$ and $\mathrm{N}$ with equation (2) and equation (3) as follows.

$$
C^{2}=10^{\frac{\alpha_{p}}{10}}-1
$$

$$
N=\frac{\lg a}{\lg \lambda_{s}}, \quad a=\sqrt{\frac{10^{\frac{\alpha_{s}}{10}}-1}{10^{\frac{\alpha_{p}}{10}}-1}}
$$

\section{B. Parameters design of Butterworth}

PROFIBUS-DP AI is mainly used in the industrial field. Industrial site there is a lot of noise signal, which is mainly composed of high-frequency noise, generally above $5000 \mathrm{~Hz}$, so the design Butterworth low-pass filter, the required cut-off frequency $f_{p}=5000 \mathrm{~Hz}$, the maximum attenuation of passband is $\alpha_{p}=3 d B$, the stopband starting frequency is $f_{s}=10000 \mathrm{~Hz}$, minimum attenuation of stopband is $\alpha_{s}=30 d B$.

$$
\Omega_{p}=2 \pi f_{p}=2 \pi \times 5000
$$

III. $\Omega_{s}=2 \pi f_{s}=2 \pi \times 10000$.

1) Use the following equation to calculate the normalized frequency. as follows:

$$
\lambda_{p}=\frac{\Omega_{p}}{\Omega_{p}}=1 \quad \lambda_{s}=\frac{\Omega_{s}}{\Omega_{p}}=2 .
$$

2) Calculate the constant $C$,with $\lambda_{p}$ and $\lambda_{s}$ in equation (2), as follows: the calculation results of $C$ is 1.

$$
C^{2}=10^{\frac{\alpha_{p}}{10}}-1=10^{0.3}-1=1 \text {. }
$$

3) Calculate the order of Butterworth,with $\alpha_{p}$ and $\alpha_{s}$ in equation (3), as follows: the calculation results that, $a$ is 31.637 and $N$ is 4.982, so the $N$ is 5 .

$$
\begin{gathered}
a=\sqrt{\frac{10^{\frac{\alpha_{s}}{10}}-1}{10^{\frac{\alpha_{p}}{10}}-1}=\sqrt{\frac{10^{3}-1}{10^{0.3}-1}}=31.637 .} \\
N=\frac{\lg a}{\lg \lambda_{s}}=\frac{\lg 31.637}{\lg 2}=4.982 .
\end{gathered}
$$

4) Look-up the normalized table of Butterworth with parameters $N$, we can get the normalized system function $H(p)$ of butterworth low-pass prototype filter, as follow: 


$$
H(p)=\frac{1}{p^{5}+3.2361 p^{4}+5.2361 p^{3}+5.2361 p^{2}+3.2361 p+1}
$$

\section{5) Remove the normalized impact.}

$$
\begin{aligned}
& H(s)=\left.H(p)\right|_{p=\frac{s}{\Omega_{p}}} \\
& =\frac{10^{20} \pi^{5}}{s^{5}+3.236 \times 10^{4} \pi s^{4}+5.236 \times 10^{8} \pi^{2} s^{3}+5.236 \times 10^{12} \pi^{3} s^{2}+3.236 \times 10^{16} \pi^{4} s+10^{20} \pi^{5}}
\end{aligned}
$$

\section{B. The structure design of Butterworth}

The 5-order Butterworth low-pass filter structure as shown in Figure 3. The 5-order parameter $\mathrm{f} 1=\mathrm{f} 2=\mathrm{f} 3=1.0$; $\mathrm{Q} 1=0.5, \mathrm{Q} 2=0.618034, \mathrm{Q} 3=1.618034$ in the normalized form of Butterworth LPF. In accordance with the standard E12 series, the $\mathrm{C} 11=\mathrm{C} 21=\mathrm{C} 31=2.2 \mathrm{nF}$. Low noise circuit design, resistance value should choose smaller. Requiring low current consumption of the occasion, the larger should be used in order to reduce the load resistor values.

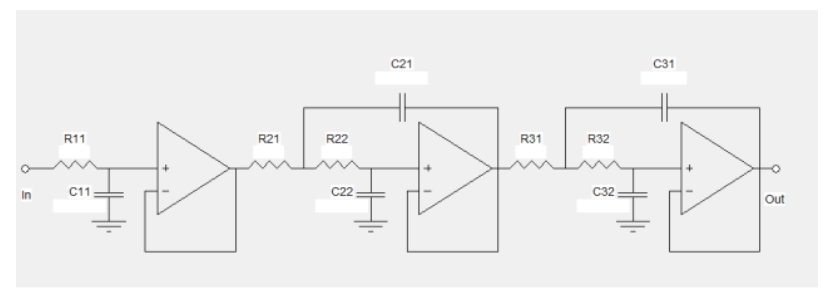

Figure 3. The parameters of Butterworth low pass filte

As follows the equation (4) and equation (5) are the $\mathrm{RC}$ circuit parameters calculation equation of Sallen-Key which is a positive feedback active filter.

$$
\begin{aligned}
\mathrm{fc} & =\frac{1}{2 \pi * \mathrm{Cf} * \mathrm{Rf}} \\
c & =2 Q^{*} \mathrm{Cf}
\end{aligned}
$$

1) Calculate the R11, with $f 1=1 k, C 11=C f=2.2 n F$ as in equation (4), the result of $R 11$ as follows:

$$
\mathrm{R} 11=\frac{1}{2 \pi * \mathrm{Cf} * \mathrm{fc}}=1 / 2 \pi * 1 \mathrm{kHz} * 2.2 \mathrm{nF}=7.234 \mathrm{k}
$$

2) Calculate the R21,R22, with $Q 2$ as in equation (5), the result as follows :Cf $=17.8 n F, C 22=14.4 n F$, than Calculate the R21 with Cf as in equation (4):

$$
\begin{gathered}
\mathrm{Cf}=2.2 \mathrm{nF} / 2 * 618034=17.8 \mathrm{nF} . \\
\mathrm{C} 22=\mathrm{Cf} / 2 \pi * 0.618034=14.4 \mathrm{nF} .
\end{gathered}
$$$$
\mathrm{R} 21=\mathrm{R} 22=\frac{1}{2 \pi * \mathrm{C} 21 * \mathrm{fc}}=1 / 2 \pi * 1 \mathrm{kHz} * 1.78 \mathrm{nF}=8.942 \mathrm{k} .
$$

3) Calculate the result of $R 32, R 32$ and C32 as follows:
$\mathrm{R} 31=\mathrm{R} 32=23.41 \mathrm{k}$

$$
\mathrm{C} 32=2.101 \mathrm{nF} \text {. }
$$

As Figure 4 shown, the amplitude frequency characteristics of the 5-order Butterworth low-pass filter which used of the calculation value of RC. The cutoff frequency of passband is $f_{p}=5000 \mathrm{~Hz}$, the attenuation is $3 \mathrm{~dB}$, the Starting frequency of stopband is $f_{s}=10000 \mathrm{~Hz}$, the attenuation is $30 \mathrm{~dB}$.

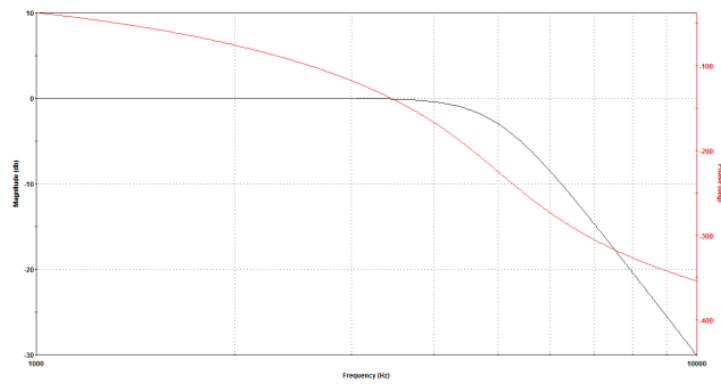

Figure 4. The amplitude frequency characteristic of Butterworth low pass filter

As Figure 5 shown, using 1-order RC filter, which could not be effectively suppress the high frequency noise, and the SNR is reduced. As Shown in Figure 6, the 5-order Butterworth filter, can effectively suppress the high-frequency signal, and detects the input signal is controlled to output the excitation signal has a smaller distortion.

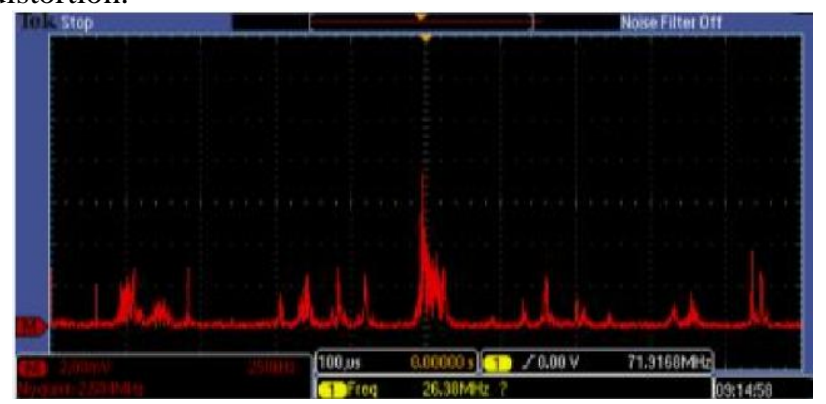

Figure 5. The 1 order RC filter characteristics of FFT

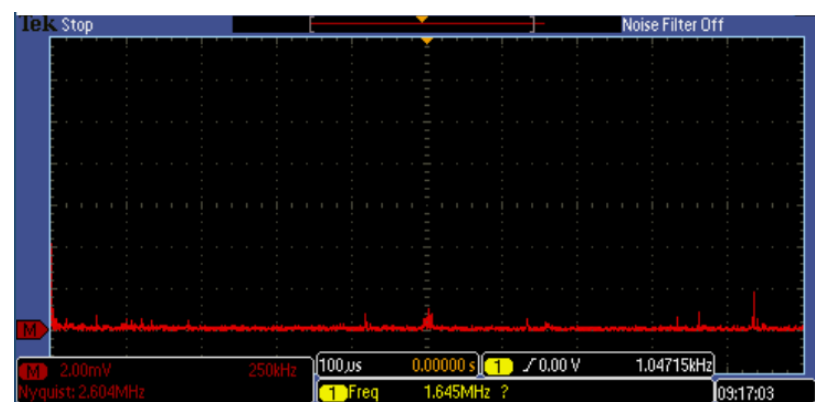

Figure 6. The 5 order Butterworth filter characteristics of FFT 


\section{CONCLUSIONS}

For the high frequency characteristics of the signal acquisition system and the noise of industrial field, this paper used 5-order Butterworth to filter more than $5 \mathrm{kHz}$ high frequency which can effectively reduce the signal interference to the acquisition system, and proposed a design of PROFIBUS-DP AI based on multi-order Butterworth low-pass filter, and gave the derivation process of filter, calculation process of $\mathrm{RC}$ value. It has been found that this scheme can effectively improve the signal-to-noise ratio of the system in industrial field, has strong control ability to suppress interference signal, and has the advantage of smaller distortion and so on to the input signal which should be measured.

\section{ACKNOWLEDGMENT}

This work has been supported by the National High-Tech Research and Development Program of China (Grant No. 2012AA040602-4).

\section{REFERENCES}

[1] Wang Z,Wang T R,Song Y Q,et al. Survey of realtime schedule theory and the state-of-the-are of ITS application in industrial real-time communication system(Part one)[ J], Information and Control,2002,21(2):146-152.)

[2] BrySon,A.E, and Y.C.HO. Applied Optimal Control, Blaisdee Walthan,Mass, 1969

[3] Richard C D. Modem control system,Addison-Wesley, Reading Massachn,1989:150 159

[4] Wang yonChu. Buttworth filter Application in process control. Industrial Instrumentation \& Automation,1994（6）: :13 15

[5] Robertson D G E DOWLING J J. Design and responses of Butterworth and critically damped digital filters, Journal of Electromyography and Kinesiology, 2003(13)

[6] Teisoku no Tameno Filter Kairo sekkei.CQ Publishing Co.,Ltd.,Tokyo. 